\title{
Association of ACE2 Polymorphisms and Derived Haplotypes With Obesity and Hyperlipidemia in Female Spanish Adolescents
}

\section{J. Lumpuy-Castillo}

IIS-FJD: Instituto de Investigacion Sanitaria de la Fundacion Jimenez Diaz

C. Vales-Villamarín

IIS-FJD: Instituto de Investigacion Sanitaria de la Fundacion Jimenez Diaz

I. Mahílo-Fernández

IIS-FJD: Instituto de Investigacion Sanitaria de la Fundacion Jimenez Diaz

\section{Pérez-Nadador}

IIS-FJD: Instituto de Investigacion Sanitaria de la Fundacion Jimenez Diaz

L. Soriano-Guillén

Fundación Jiménez Díaz: Hospital Universitario Fundacion Jimenez Diaz

Oscar Lorenzo ( $\nabla$ olorenzo@fjd.es )

IIS-Fundación Jiménez Díaz-UAM https://orcid.org/0000-0001-5515-6078

C. Garcés

IIS-FJD: Instituto de Investigacion Sanitaria de la Fundacion Jimenez Diaz

\section{Research Article}

Keywords: ACE2, SNP, haplotype, cardiovascular, obesity, hyperlipidemia

Posted Date: October 19th, 2021

DOl: https://doi.org/10.21203/rs.3.rs-968623/v1

License: (9) This work is licensed under a Creative Commons Attribution 4.0 International License. Read Full License 


\section{Abstract}

Background: In the cardiovascular (CV) system, overactivation of the angiotensin converting enzyme (ACE) may trigger deleterious responses derived from angiotensin (Ang)-II, which can be attenuated by stimulation of ACE2 and subsequent Ang-(1-7) metabolite. However, ACE2 exhibits a high degree of genetic polymorphism that may affect its structure and stability, interfering with these cardioprotective actions.

Methodology: Five ACE2-single nucleotide polymorphisms (SNP); rs4646188, rs2158083, rs233575, rs879922, and rs2074192, previously related with CV risk factors, were analyzed in a general population of adolescents and tested for potential associations with anthropometric and plasma parameters.

Results: Girls ( $n=461)$ exhibited lower rates of overweight and obesity, blood pressure, and glycemia than boys $(n=412)$, though increased plasma lipids. The triglycerides (TG)/HDL-C ratio was, however, lower in females. Interestingly, only in girls, the occurrence of overweight/obesity was associated with the SNPs rs879922 [OR 1.67 (1.02-2.75)], rs233575 [OR 1.98 (1.21-3.22)] and rs2158083 [OR 1.67 (1.04-2.68)]. Also, their highest levels of TG were linked to rs879922, rs233575, and rs2158083, and the highest TG/HDL-C ratio was assocated with rs879922 and rs233575. The upper levels of TC and LDL-C was associated with rs2074192 and rs2158083. Furthermore, the established cut-off level for TG $\geq 90 \mathrm{mg} / \mathrm{dl}$ was related with rs879922 [OR 1.78 (1.06-2.96)], rs2158083 [OR 1.75 (1.08-2.82)], and rs233575 [OR 1.62 $(1.00$-2.61)]. The cut-off level for TC $\geq 170 \mathrm{mg} / \mathrm{dl}$ was associated with rs2074192 [OR 1.54 (1.04-2.28) and rs2158083 [OR 1.53 (1.04-2.25)]. In addition, the haplotype (C-G-C) derived from rs879922rs2158083-rs233575 was related with higher prevalence of overweight/obesity and TG elevation.

Conclusion: The expression and activity of ACE2 may be essential for CV homeostasis. Interestingly, ACE2-SNPs rs879922, rs233575, rs2158083 and rs2074192, and haplotype (C-G-C) of the three former could induce vulnerability to obesity and hyperlipidemia in women. Thus, these SNPs might be used as predictive biomarkers for CV diseases and as molecular targets for CV therapy.

\section{Introduction}

The Angiotensin converting enzyme-2 (ACE2) is a significant regulator of the Renin-AngiotensinAldosterone system (RAAS), which plays key roles in the control of cardiovascular (CV) system [1]. In the canonical pathway, the RAAS precursor, angiotensinogen, is degraded to the octapeptide angiotensin-II (Ang-II) by consecutive digestions of renin and angiotensin convertase enzyme (ACE). Ang-ll could be then converted by aminopeptidase-A to Ang-III, which conserves similar proprieties mostly mediated by two distinct G protein-coupled receptors named AT1R and AT2R [2]. Overactivation of the ACE/AngII/AT1R pathway has been associated with several CV pathologies, including hypertension, heart failure, vascular inflammation and remodelling, coagulation, and atherosclerosis [3]. However, activation of AT2R can trigger anti-inflammation and vasodilation. In this regard, the stimulation of the non-canonical axis of the RAAS may compensate ACE/Ang-II/AT1R actions. The mono-carboxypeptidase ACE2 can convert 
Ang-II into angiotensin 1-7 (Ang (1-7)), which binds to the Mas receptor (MasR) [1]. The ACE2/Ang (17)/MasR axis is able to reduce blood pressure and CV hypertrophy and fibrosis, stimulating vasoactive prostaglandins and lessening redox imbalance [4]. Ang (1-7) also protects ACE-degradation of bradykinins and ameliorates inflammatory responses, and vascular permeability [1]. Moreover, ACE2 also degrades Ang I into Ang (1-9), decreasing Ang-II levels. Given the opposite effects of Ang (1-7) and Ang-II in the CV homeostasis, it might be imperative to maintain a minimum ACE2 level or activity.

Interestingly, the ACE2 gene is highly conserved in mammals and expressed in testes, renal and CV system, and especially in gastrointestinal tissues [5]. It encodes a type I membrane-bounding glycoprotein composed by 805 amino acids divided in three functional domains including a C-terminal transmembrane anchoring region, a N-terminal signal peptide motif, and an HEXXH zinc-binding metalloprotease region (Figure 1). ACE2 maps in chromosome Xp22 and contain 17 introns and 18 exons, where a high degree of genetic polymorphism can be found [6]. There are at least 510 valid variants in ACE2 (mostly intronic regions) and particularly [7], some single nucleotide polymorphisms (SNP) have been associated with CV risk factors. Genetic variations in ACE2 may affect its structure and stability, interfering with its cardioprotective functions. In adults with different gender, ethnic and presence of pre-existing CV disease, rs4646188, rs2158083, rs233575, rs879922, and rs2074192 SNPs (Figure 1) have been related with hypertension, ventricular hypertrophy or type-2 diabetes mellitus (T2DM), and increased plasma total cholesterol (TC), triglycerides (TG), low-density lipoproteincholesterol (LDL-C), or reduced high-density lipoprotein-cholesterol (HDL-C) and Ang (1-7) [8-11]. In children or adolescent cohorts, where existence of confusing factors (i.e., epigenetic alterations) is lower, boys but not girls showed association of rs2158083, rs233575 and rs2074192 with hypertension [12][13]. Nowadays, regulation of the ACE2 expression and activity has become essential for Coronavirus disease (COVID)-19 subjects, particularly for those with cardiovascular complications [6][14]. Thus, further studies analysing ACE2-SNPs and related haplotypes in young populations, could suggest predictive markers for $\mathrm{CV}$ diseases and molecular targets for $\mathrm{CV}$ therapy.

\section{Methodology}

\section{Subjects}

The present study comprised 873 adolescents (12-16 years old) recruited in the study "Four Provinces" during 2004-08 in whom information on biochemical variables and DNA samples were available [15]. This cross-sectional study was designed to analyze CV risk factors in general population of adolescents from Spain (461 girls and 412 boys). Children suffering from any endocrine, metabolic, hepatic, or renal disorder were excluded from the study. Specialized physicians and nurses oversaw the anthropometric characterization and blood extractions. The investigation fulfils the principles contained in the Declaration of Helsinki and subsequent reviews, as well as the prevailing Spanish legislation on clinical research in human subjects. Parents were required to sign a written consent form allowing their children to participate. The study protocol was approved by the Ethics Committee of Clinical Investigation of the Fundación Jiménez Díaz (Code number: PIC016-2019 FJD). 
Variables of the Study

\section{i) Anthropometric and blood pressure measurements}

The weight and height were taken in barefoot children, wearing light clothing. Both, weight, and height were approached to the nearest 0.1 unit ( $\mathrm{kg}$ or $\mathrm{cm}$, respectively) and were used to estimate the body mass index (BMI), as kilograms divided by height in squared meters $\left(\mathrm{kg} / \mathrm{m}^{2}\right)$. The age- and sex-specific cut-off points of BMI for overweight and obesity were established according to Cole et al [16]. In addition, the blood pressure (systolic and diastolic) was quantified in subjects as average of two measurements in the right arm by using a mercury sphygmomanometer and after 15 min resting in seated position.

\section{ii) Plasma parameters}

Blood samples were obtained from subjects in the morning after a 12-h fasting period by using EDTAcollection tubes (Vacutainer, BD). Plasma was separated by centrifuging blood at $1,500 \mathrm{~g}$ at $4^{\circ} \mathrm{C}$ for 20 min. The upper fraction was stored at $-80^{\circ} \mathrm{C}$ until use. Total cholesterol and triglycerides were enzymatically quantified in a RA-1000 Autoanalyzer (Technicon, Luton, UK). HDL-C concentrations were measured after precipitation of apo B-containing lipoproteins with phosphotungstic acid and $\mathrm{Mg}^{++}$ (Boehringer Mannheim, Baden-Wurttemberg, Germany). LDL-C levels were calculated according to Friedewald's formula. Also, the glucose concentration was evaluated by the glucose oxidase method, and insulin levels were achieved by using a RIA commercial kit (BI-Insulin IRMA, Bio-Rad, France). To estimate insulin resistance, the homeostatic model assessment of insulin resistance (HOMA-IR) index was calculated following formula: fasting insulin $(\mu \mathrm{U} / \mathrm{ml}) \times$ fasting glucose $(\mathrm{mmol} /$ liter $) / 22.5$.

\section{iii) Single nucleotide polymorphism (SNPs) determinations}

Genomic DNA was also obtained from blood samples. After plasma separation, the interphasic fraction (leukocytes) was used to isolate total DNA by adaptation of a classic procedure, which involves salting out of the cellular proteins by dehydration and precipitation with a saturated $\mathrm{NaCl}$ solution [17]. The quantity and quality of DNA was assessed by UV-spectrophotometry using the Nanodrop spectrophotometer ND-1000. Five SNPs located in intronic sequences of the ACE2 gene (A/G [rs4646188], G/C [rs879922], A/G [rs233575], C/T [rs2074192] and T/C [rs2158083]) were genotyped by using predesigned TaqMan® SNP Genotyping Assays from Applied Biosystems (C_32336232_10, C_8877953_10, C_2260459_10, C_16163821_10 and C_16141434_10, respectively). A StepOnePlus ${ }^{\text {TM }}$ Real-Time PCR System (Applied Biosystems) was used for allelic discrimination. PCR was performed with a mixture of $10 \mathrm{ng}$ of genomic DNA, TaqMan® SNP Genotyping Assay (20X), and TaqPath ${ }^{\text {TM }}$ ProAmp ${ }^{\mathrm{TM}}$ Master Mix (Applied Biosystems). Samples were cycled under the recommended conditions: $95^{\circ} \mathrm{C}$ for $10 \mathrm{~min}, 95^{\circ} \mathrm{C}$ for $15 \mathrm{sec}$ and $60^{\circ} \mathrm{C}$ for $1 \mathrm{~min}$, repeated over 40 cycles.

\section{iv) Haplotype analysis}

The five SNPs (rs4646188, rs879922, rs233575, rs2074192, and rs2158083) were selected to construct haplotypes. Then, the haplo.glm function from the haplo.stats package was used for haplotype analysis. Haplotypes with frequencies $<5 \%$ in the population were not considered. 


\section{Statistical analysis}

Qualitative variables were included as absolute and relative frequencies. Associations between qualitative variables were studied by the chi-square test and odds ratio. On the other hand, normality of quantitative variables was analyzed by the Kolmogorov-Smirnov test. On one hand, variables with normal distribution (age and HDL-C) were compared using a Student's t-test and ANOVA one way, followed by Tukey (assuming equal variances) or Games-Howell (equal variances not assumed) post-hoc test. Also, they were summarized as mean values and $95 \%$ confidence interval $(\mathrm{Cl})$. Some variables with non-normal distribution were normalized by log base-10 transformation [systolic (SBP) and diastolic (DBP) blood pressures, TC, TG, LDL-C, TG/HDL] and were expressed as geometric mean values and $95 \%$ confidence interval. Other variables with non-normal distribution (glucose, insulin, and HOMA-IR) were compared using the Mann-Whitney U test and Kruskal-Wallis test followed by Dunn's (post-Hoc). These variables were summarized by median and interquartile range. The associations of haplotypes with anthropometric, blood pressure and plasma parameters were analysed by regression models. For continuous variables, we used linear models, whereas dichotomous coded variables were examined by logistic models. The former were summarized by coefficients (coef.) while the later were summarized by odds ratio (OR). Also, a 95\% $\mathrm{Cl}$ and p-values were reported. Statistical analyses were performed using the statistical language R version 4.0.5 (R Foundation for Statistical Computing, Vienna, Austria).

\section{Results}

\section{Characterization of the population}

We analysed a population of 873 adolescents aged 13.8 years with a mean BMI of $21.5 \mathrm{~kg} / \mathrm{m}^{2}$. Following the established definition for child overweight and obesity, $71.8 \%$ of them were normo-weight whereas $28.2 \%$ showed an excessive weight (Table 1$)$. The mean BMI Z-score was $0.00(-0.067-0.067)$ for the global population, and $-0.018(-0.10-0.07)$ and 0.021 (-0.08-0.12), for girls and boys, respectively $(p=0.569)$. Also, according to the latest clinical guides of the American College of Cardiology/American Heart Association/American Diabetes Association for paediatric population [18][19], they were, on average, in the normotensive range and showed unaltered levels of the glycemic and lipid profiles (Table 1). However, further analysis in females and males pointed out significant differences between sex. Girls (52.8\%) exhibited lower rates of overweight and obesity, SBP, and glycemia, but higher levels of TC, LDL-C, and HDL-C than boys (Table 1). The ratio of TG and HDL-C, as a predictor of metabolic syndrome and CV diseases [20], was significantly lower in female individuals.

\section{Genotypic and allelic frequencies of ACE2 SNPs}

We next analyzed five single nucleotide polymorphisms (SNPs) of the ACE2 gene related with CV injuries [21][9][10]. Firstly, the genotype frequencies of rs4646188, rs879922, rs233575, rs2074192, and rs2158083 were found in a Hardy's-Weinberg equilibrium. The prevalence of the minor alleles of the studied SNPs in girls and boys, respectively, were: (G) in rs4646188, 10.1\% and 8.4\%; (C) in rs879922, 
42.4\% and 39.3\%; (G) in rs233575, 39.2\% and 37.3\%; (T) in rs2074192, 40.10\% and $41.5 \%$, and $(\mathrm{C})$ in rs2158083, 38.45\% and 36.3\% (Table 2).

\section{Association between ACE2 SNPs and overweight and obesity}

Next, we tested whether these five ACE2 SNPs (rs4646188, rs879922, rs233575, rs2074192, and rs2158083) might associate with the anthropomorphic variables of the population. Remarkably, none of the SNPs were significantly related with the SBP and DBP, either in girls or boys (not shown). However, in females, the occurrence of overweight or obesity was significantly associated with three of the studied SNPs (Table 3). In particular, the minor allele $C$ of the rs 879922 variant was present in $26.9 \%$ of overweight or obese females [OR 1.67 (95\% Cl: 1.02-2.75), $p=0.042$ ]. Similarly, the G allele of rs 233575 and the $C$ allele of rs 2158083 were present in $28.0 \%$ [OR 1.98 (1.21-3.22), $p=0.006$ ] and $27.0 \%$ [OR 1.67 (1.04-2.68), $p=0.032$ ] of overweight or obese girls, respectively (Table 3 ). In contrast, the overweight or obesity condition was not related with any SNPs in the male population (not shown). Thus, the presence of rs879922, rs233575, and rs2158083, previously related with CV damages [21][9][10], were also associated with overweight and obesity in adolescent females.

\section{Association between ACE2 SNPs and the glucose and lipid profiles}

Further potential relationships between the SNPs and biochemical variables were also assessed. Glycemic parameters such as glycemia, plasma insulin and the HOMA-IR were not significantly associated with the presence of any SNPs in both sexes (not shown). Lipid parameters did not show any relationship with SNPs in male adolescents. However, again in females, four out of five SNPs were linked with the lipid profile. The highest levels of TG were significantly associated with the presence of heterozygous genotypes of rs879922 ( $p=0.020)$, rs233575 ( $p=0.017)$ and rs2158083 ( $p=0.036)$ (Table 4). The TG/HDL-C ratio also linked with rs879922 and rs233575. In addition, the established cut-off levels of TG (over $90 \mathrm{mg} / \mathrm{dl}$; 75th percentile) for adolescents (10-19 years-old) [18] were related with rs879922 [OR 1.78 (95\% Cl: 1.06-2.96)], rs2158083 [OR 1.75 (1.08-2.82)], and rs233575 [OR 1.61 (1.00-2.62)] (Figure $2 A)$. Also, the highest levels of TC and LDL-C were related with the heterozygous genotypes of rs2074192 $(p=0.003$ and $p=0.03$, respectively) and $r 2158083(p=0.008$ and $p=0.019$, respectively) (Table 4). Similarly, the cut-off levels of TC (over $170 \mathrm{mg} / \mathrm{dl}$; 75th percentile), but not that of LDL-C levels, for adolescents (10-19 years-old) [18] were associated with rs2074192 [OR 1.54 (95\% Cl: 1.04-2.28) and rs2158083 [OR 1.53 (1.04-2.25)] (Figure 2B). The HDL-C levels were, however, independent of the existence of these ACE2 SNPs. Therefore, the presence of ACE2 SNPs rs879922, rs233575 and rs2158083 may be useful to predict elevated levels of TG and TC in girls.

\section{Association between ACE2-haplotypes and overweight/obesity and TG levels}

Since rs879922, rs233575, rs2074192, and rs2158083 were related with overweight/obesity and lipid alterations in females, we examined whether combinations of these SNPs might also associate with higher risk of both pathologies. In particular, the haplotype composed by minor alleles of rs879922, rs233575, and rs2158083 (C-G-C) was overrepresented in girls (34\%) and linked to significant higher BMI 
[coef. $0.01(0.001,0.019 ; 95 \% \mathrm{Cl}), \mathrm{p}=0.038$ ], in comparison with the haplotype composed by their major alleles (G-A-T) (Table 5). The C-G-C haplotype was also associated with the presence of overweight/obesity [OR $1.41(1.01,1.97 ; 95 \% \mathrm{Cl}), \mathrm{p}=0.044$ ], and elevated plasma TG [coef. $0.023(0.002$, 0.044; $95 \% \mathrm{Cl}), \mathrm{p}=0.031$ ] and TG/HDL ratio [(coef. $0.031(0.001,0.061 ; 95 \% \mathrm{Cl}), \mathrm{p}=0.045]$. Interestingly, after adjusting by $\mathrm{BMI}$, the $\mathrm{C}-\mathrm{G}-\mathrm{C}$ haplotype maintained its association with higher TG levels [coef. 0.023 $(0.002,0.04495 \% \mathrm{Cl}), p=0.034]$ (Table 5). On the other hand, the haplotype composed by minor alleles of rs22074192 and rs2158083 (C-C) was overrepresented in 37\% subjects but did not significantly associate with either BMI or lipid levels (not shown). Thus, C-G-C haplotype of rs879922, rs233575, and rs2158083 SNPs could be considered a risk marker for obesity and dyslipemia in females.

\section{Discussion}

In this work we have analyzed five SNPs in the ACE2 gene in a general population of apparently healthy adolescents from Spain. The prevalence of these polymorphisms in females and males was comparable to previous findings $[12,22,23]$, but the occurrence of some of them was associated with CV risk factors only in girls.

At these ages, females showed lower rates of overweight and obesity, SBP and glycemia than males. Although girls showed higher TC and LDL-C, they enhanced HDL-C, as compensation. They also exhibited lower TG/HDL ratio, which is a predictor of metabolic syndrome and CV diseases (i.e., coronary artery disease or atherosclerosis) and an estimator of LDL-C particle size [20]. Thus, in principle, young girls may be cardio-protected, as previously described [24][25]. Spite of the differences in hormonal regulation between sexes [26], a potential explanation of this cardio-protection could be based on the expression of ACE2 and subsequent effects on the RAAS activity. Since ACE2 is encoded in chromosome $\mathrm{X}$, and specifically in a region that escape from X-inactivation, ACE2 may be upregulated in girls [27]. Then, the ACE2/Ang (1-7)/MasR activation could preserve pancreas, skeletal muscle, adipose tissue or the CV system from oxidation, inflammation, and fibrosis [28]. Moreover, the elevation of ACE2 involve a reduction of Ang-II, which enhances the NADH/NADPH oxidase activity and reduces the nitric oxidedependent vasodilatation at systemic level [29]. Also, Ang-II stimulates cholesterol biosynthesis, overexpresses lipid receptors (i.e., LRP1, LOX-1), facilitates the uptake and oxidation of LDL-C, and induces pro-inflammatory matrix proteins and matrix-degrading enzymes (i.e., MCP-1, IL-18, PAI-1) [30] [31]. In addition, ACE2 may defend the CV system by direct actions. ACE2 increases mitochondrial ATP production and reduces pro-oxidant factors such as NOX4. It also ameliorates lipid deposition and endoplasmic reticule stress via GRP78/elF2a/XBP-1/ATF4/CHOP expression [32].

Importantly, a reduction of ACE2 levels and subsequent Ang (1-7)-MasR signalling can promote CV failures [33]. ACE2 knockout mice showed increased lipid accumulation, ER stress and mitochondrial dysfunction in skeletal muscle, and overexpression of ACE2 reduced these responses and improved glucose and lipid metabolism through the IKKB/NFkB/IRS-1 pathway [34][32]. Similarly, MasR knockout exhibited dyslipemia and insulin resistance, along with decreased adiponectin secretion and glucose uptake [35]. Furthermore, during hypertension or diabetes, a deficiency of ACE2 and increased ACE/ACE2 
ratio have been noted [36], and cardioprotective treatments with ACE inhibitors and Ang-II receptor blockers exert their effects partly by increasing ACE2 levels [37]. In this regard, we propose that in those girls who carried specific intergenic ACE2 SNPs, the integrity, activity and/or stability of the ACE2 RNAmessenger and resultant protein could have been affected [38][39], and Ang-II signalling may overcome those of ACE2/Ang (1-7)/MasR. In fact, the existence of rs879922, rs233575, and rs2158083 SNPs of ACE2 was associated with overweight/obesity and elevated plasma TG in Spanish girls. The rs 879922 and rs233575 polymorphisms were also related with upper TG/HDL-C ratio, and the rs2074192 and rs2158083 with higher TC levels. Reinforcing our findings, we observed that the C-G-C haplotype of rs879922, rs233575, and rs2158083 was associated with excessive weight and elevated TG levels. Previous data on ACE2 haplotypes related higher risk of cardiac hypertrophy with minor alleles of rs2074192 and rs2106809 in females [10] or with those of rs2106809 and rs6632677 in males [40].

Little is known about rs2158083 and rs233575. The former is encoded in ACE2's intron 4 and was previously associated with higher blood pressure [12]. Similarly, rs233575 (intron 16) was related with higher blood pressure in adolescents and its interaction with obesity was present only in females [13]. It was also linked to increased LDL-C ( $\geq 1.8 \mathrm{mmol} / \mathrm{L})$ [8] and T2DM [11], and associated with higher LVMI [41]. The rs233575 SNP may alter binding of regulatory factors such as RNA-binding proteins involved in splicing (e.g., ARID5A), which is stimulated under inflammation [42]. Moreover, rs879922 (intron 11) can be considered a significant quantitative trait locus for essential hypertension in female Chinese, where it also associated with increased TC ( $\geq 5.2 \mathrm{mmol} / \mathrm{L})$ and LDL-C ( $\geq 1.8 \mathrm{mmol} / \mathrm{L})$ [8]. rs879922 was linked with T2DM [11] and cardiac hypertrophy [41], and importantly, the relative expression of ACE2 was significantly lower in subjects with the CC genotype of rs879922 compared with those carrying the GG/CG genotype [43]. Finally, rs2074192 (intron 16) has been associated with CV risk (i.e., LVH, carotid arteriosclerosis stenosis, microalbuminuria), retinopathy in T2DM individuals, hypertension and cardiac hypertrophy [10-12]. rs2074192 was also linked to increased TC ( $\geq 5.2 \mathrm{mmol} / \mathrm{L})$ in hypertensive population [8], and neonates with rs2074192 were more likely to be born as small for gestational age babies, which contribute to metabolic syndrome and CV diseases in later life [44]. Interestingly, rs2074192 was associated with reduced circulating Ang (1-7) levels [22], and in COVID-19 patients, rs2074192 correlated with more severe outcomes (i.e., bilateral pneumonia, dyspnea, high fever) [45][13].

\section{Limitations Of The Study}

At these ages, girls and boys may produce different sexual hormones and factors, which can influence on the lipid profile. Thus, their puberal stage may add important information to explain differences between sex. Also, the precise biological mechanism and other possible factors underlying the association of the ACE2 gene with overweight/obesity or plasma lipids elevation remain to be clarified. Finally, functional

investigations of the association between C-G-C haplotype with overweight/obesity or TG elevation could be required.

\section{Conclusion}


The ACE2 gene may become a double-sword factor for CV protection, at least in women. Those female adolescents who carry the wild-type ACE2 gene may be somehow protected from increased adiposity and hyperlipidemia. However, Spanish girls carrying the rare alleles of the ACE2-SNPs rs879922, rs233575, rs2158083, or rs2074192 may be vulnerable to future obesity and CV injuries. The haplotype rs879922rs233575-rs2158083 may be considered a valid biomarker for both pathologies. Therefore, these ACE2SNPs might be addressed for therapeutic and prognostic purposes against CV diseases. In this sense, new studies might evaluate their role as predictors of worse evolution in COVID-19 subjects, where CV homeostasis may be seriously damaged.

\section{Abbreviations}

ACE, angiotensin convertase enzyme

ACE2, Angiotensin converting enzyme 2

Ang (1-7), angiotensin 1-7

Ang-Il, angiotensin-II

ARID5A, AT-Rich Interaction Domain 5A

COVID, Coronavirus disease

GRP78/elF2a/XBP-1/ATF4/CHOP, Glucose-Regulated Protein,78-KD/Eukaryotic Initiation Factor 2/X-box binding protein 1/C/EBP Homologous Protein

HDL-C, high-density lipoprotein-cholesterol

IKKß/NFkB/IRS-1, IKB Kinase-beta/nuclear factor-kappa B/insulin receptor substrate 1

IL-18, interleukin-18

LDL-C, low-density lipoprotein-cholesterol

LOX-1, lectin-like OxLDL receptor 1

LRP1, LDL Receptor Related Protein 1

MasR, Mas receptor

MCP-1, Monocyte chemoattractant protein-1

$\mathrm{NAD}(\mathrm{P}) \mathrm{H}$, nicotinamide adenine dinucleotide (phosphate) hydrogen

PAl-1, Plasminogen activator inhibitor-1 
RAAS, Renin-Angiotensin-Aldosterone system

T2DM, type-2 diabetes mellitus

TC, total cholesterol

TG, triglycerides

\section{Declarations}

\section{Ethics approval and consent to participate}

The investigation fulfils the principles contained in the Declaration of Helsinki and subsequent reviews, as well as the prevailing Spanish legislation on clinical research in human subjects. Parents were required to sign a written consent form allowing their children to participate. The study protocol was approved by the Ethics Committee of Clinical Investigation of the Fundación Jiménez Díaz (Code number: PIC0162019 FJD).

\section{Competing interests}

The authors declare that they have no competing interests

\section{Funding}

This research was funded by the Fondo de Investigación Sanitaria-IS. Carlos III (grant numbers PI18/01016 and PI20/00923), Biobank (grant number FEDERRD09/0076/00101), and Ciberdem (reference: CB07/08/2007).

\section{Consent for publication}

Not applicable

\section{Availability of data and materials}

Not applicable

\section{Authors' contributions}

L-C J., V-V C. and P-N I. quantified the gene expression of ACE2-SNPs and analyze its associations with clinical variables. M-F I. performed the statistic studies. S-G L., L O. and G C. designed and wrote the manuscript. All authors participate in the discussion of the work

\section{Acknowledgements}

The article is dedicated to the late Prof. Manuel de Oya as the warmest homage to his memory. 


\section{References}

1. Santos RAS, Sampaio WO, Alzamora AC, Motta-Santos D, Alenina N, Bader M, et al. The ACE2/Angiotensin-(1-7)/MAS Axis of the Renin-Angiotensin System: Focus on Angiotensin-(1-7). Physiol Rev. 2018;98:505-53.

2. Jugdutt BI. Expanding Saga of the Renin-Angiotensin System. Circulation American Heart Association. 2015;131:1380-3.

3. Sriram K, Insel PA. A hypothesis for pathobiology and treatment of COVID-19: The centrality of ACE1/ACE2 imbalance. Br J Pharmacol. 2020. doi:10.1111/bph.15082.

4. Povlsen AL, Grimm D, Wehland $M$, Infanger $M$, Krüger $M$. The Vasoactive Mas Receptor in Essential Hypertension. J Clin Med [Internet]. 2020 [cited 2020 Jul 9];9. Available from: https://www.ncbi.nlm.nih.gov/pmc/articles/PMC7019597/.

5. The protein expression profile of ACE2 in human tissues. Mol Syst Biol. John Wiley \& Sons, Ltd; 2020;16:e9610.

6. Lima RS, Rocha LPC, Moreira PR. Genetic and epigenetic control of ACE2 expression and its possible role in COVID-19. Cell Biochem Funct. 2021;39:713-26.

7. Curtis D. Variants in ACE2 and TMPRSS2 Genes Are Not Major Determinants of COVID-19 Severity in UK Biobank Subjects. Hum Hered. 2020;85:66-8.

8. Pan Y, Wang T, Li Y, Guan T, Lai Y, Shen Y, et al. Association of ACE2 polymorphisms with susceptibility to essential hypertension and dyslipidemia in Xinjiang, China. Lipids Health Dis. 2018;17:241.

9. Fan X, Wang Y, Sun K, Zhang W, Yang X, Wang S, et al. Polymorphisms of ACE2 gene are associated with essential hypertension and antihypertensive effects of Captopril in women. Clin Pharmacol Ther. 2007;82:187-96.

10. Fan Z, Wu G, Yue M, Ye J, Chen Y, Xu B, et al. Hypertension and hypertensive left ventricular hypertrophy are associated with ACE2 genetic polymorphism. Life Sci. 2019;225:39-45.

11. Liu C, Li Y, Guan T, Lai Y, Shen Y, Zeyaweiding A, et al. ACE2 polymorphisms associated with cardiovascular risk in Uygurs with type 2 diabetes mellitus. Cardiovasc Diabetol. 2018;17:127.

12. Malard L, Kakinami L, O'Loughlin J, Roy-Gagnon M-H, Labbe A, Pilote L, et al. The association between the Angiotensin-Converting Enzyme-2 gene and blood pressure in a cohort study of adolescents. BMC Med Genet. 2013;14:117.

13. Hamet P, Pausova Z, Attaoua R, Hishmih C, Haloui M, Shin J, et al. SARS-CoV-2 Receptor ACE2 Gene Is Associated with Hypertension and Severity of COVID 19: Interaction with Sex, Obesity, and Smoking. Am J Hypertens. 2021;34:367-76.

14. Bader M, Turner AJ, Alenina N. ACE2, a multifunctional protein - from cardiovascular regulation to COVID-19. Clin Sci Lond Engl 1979. 2020;134:3229-32.

15. de Oya I, Schoppen S, Lasunción MA, Lopez-Simon L, Riestra P, de Oya M, et al. Sex hormone-binding globulin levels and metabolic syndrome and its features in adolescents. Pediatr Diabetes. 
2010;11:188-94.

16. Cole TJ, Bellizzi MC, Flegal KM, Dietz WH. Establishing a standard definition for child overweight and obesity worldwide: international survey. BMJ. 2000;320:1240-3.

17. Miller SA, Dykes DD, Polesky HF. A simple salting out procedure for extracting DNA from human nucleated cells. Nucleic Acids Res. 1988;16:1215.

18. Grundy SM, Stone NJ, Bailey AL, Beam C, Birtcher KK, Blumenthal RS, et al 2018 AHA/ACC/AACVPR/AAPA/ABC/ACPM/ADA/AGS/APhA/ASPC/NLA/PCNA Guideline on the Management of Blood Cholesterol: A Report of the American College of Cardiology/American Heart Association Task Force on Clinical Practice Guidelines. Circulation. 2019;139:e1082-143.

19. American Diabetes Association. 13. Children and Adolescents: Standards of Medical Care in Diabetes-2020. Diabetes Care. 2020;43:S163-82.

20. Cordero A, Andrés E, Ordoñez B, León M, Laclaustra M, Grima A, et al. Usefulness of triglycerides-tohigh-density lipoprotein cholesterol ratio for predicting the first coronary event in men. Am J Cardiol. 2009;104:1393-7.

21. Itoyama S, Keicho N, Hijikata M, Quy T, Phi NC, Long HT, et al. Identification of an alternative 5'untranslated exon and new polymorphisms of angiotensin-converting enzyme 2 gene: lack of association with SARS in the Vietnamese population. Am J Med Genet A. 2005;136:52-7.

22. Chen YY, Zhang P, Zhou XM, Liu D, Zhong JC, Zhang CJ, et al. Relationship between genetic variants of ACE2 gene and circulating levels of ACE2 and its metabolites. J Clin Pharm Ther. 2018;43:18995.

23. Patel SK, Wai B, Ord M, Maclsaac RJ, Grant S, Velkoska E, et al. Association of ACE2 genetic variants with blood pressure, left ventricular mass, and cardiac function in Caucasians with type 2 diabetes. Am J Hypertens. 2012;25:216-22.

24. Mercuro G, Deidda M, Piras A, Dessalvi CC, Maffei S, Rosano GMC. Gender determinants of cardiovascular risk factors and diseases. J Cardiovasc Med Hagerstown Md. 2010;11:207-20.

25. Barstad LH, Júlíusson PB, Johnson LK, Hertel JK, Lekhal S, Hjelmesæth J. Gender-related differences in cardiometabolic risk factors and lifestyle behaviors in treatment-seeking adolescents with severe obesity. BMC Pediatr. 2018;18:61.

26. Kittnar 0 . Selected sex related differences in pathophysiology of cardiovascular system. Physiol Res. 2020;69:21-31.

27. Tukiainen T, Villani A-C, Yen A, Rivas MA, Marshall JL, Satija R, et al. Landscape of X chromosome inactivation across human tissues. Nature. 2017;550:244-8.

28. Cao X, Song L-N, Yang J-K. ACE2 and energy metabolism: the connection between COVID-19 and chronic metabolic disorders. Clin Sci Lond Engl 1979. 2021;135:535-54.

29. St Paul A, Corbett CB, Okune R, Autieri MV. Angiotensin II, Hypercholesterolemia, and Vascular Smooth Muscle Cells: A Perfect Trio for Vascular Pathology. Int J Mol Sci. 2020;21:E4525. 
30. Sendra J, Llorente-Cortés V, Costales P, Huesca-Gómez C, Badimon L. Angiotensin II upregulates LDL receptor-related protein (LRP1) expression in the vascular wall: a new pro-atherogenic mechanism of hypertension. Cardiovasc Res. 2008;78:581-9.

31. Zhang F, Hu Y, Xu Q, Ye S. Different effects of angiotensin II and angiotensin-(1-7) on vascular smooth muscle cell proliferation and migration. PloS One. 2010;5:e12323.

32. Cao X, Lu X-M, Tuo X, Liu J-Y, Zhang Y-C, Song L-N, et al. Angiotensin-converting enzyme 2 regulates endoplasmic reticulum stress and mitochondrial function to preserve skeletal muscle lipid metabolism. Lipids Health Dis. 2019;18:207.

33. Rabelo LA, Todiras M, Nunes-Souza V, Qadri F, Szijártó IA, Gollasch M, et al. Genetic Deletion of ACE2 Induces Vascular Dysfunction in C57BL/6 Mice: Role of Nitric Oxide Imbalance and Oxidative Stress. PloS One. 2016;11:e0150255.

34. Xuan X, Gao F, Ma X, Huang C, Wang Y, Deng H, et al. Activation of ACE2/angiotensin (1-7) attenuates pancreatic $\beta$ cell dedifferentiation in a high-fat-diet mouse model. Metabolism. 2018;81:83-96.

35. Santos SHS, Fernandes LR, Mario EG, Ferreira AVM, Pôrto LCJ, Alvarez-Leite Jl, et al. Mas deficiency in FVB/N mice produces marked changes in lipid and glycemic metabolism. Diabetes. 2008;57:3407.

36. Srivastava P, Badhwar S, Chandran DS, Jaryal AK, Jyotsna VP, Deepak KK. Imbalance between Angiotensin II - Angiotensin (1-7) system is associated with vascular endothelial dysfunction and inflammation in type 2 diabetes with newly diagnosed hypertension. Diabetes Metab Syndr. 2019;13:2061-8.

37. Kriszta G, Kriszta Z, Váncsa S, Hegyi PJ, Frim L, Erőss B, et al. Effects of Angiotensin-Converting Enzyme Inhibitors and Angiotensin Receptor Blockers on Angiotensin-Converting Enzyme 2 Levels: A Comprehensive Analysis Based on Animal Studies. Front Pharmacol. 2021;12:254.

38. Wysocki J, Ye M, Soler MJ, Gurley SB, Xiao HD, Bernstein KE, et al. ACE and ACE2 activity in diabetic mice. Diabetes. 2006;55:2132-9.

39. Zaina S, Pérez-Luque EL, Lund G. Genetics Talks to Epigenetics? The Interplay Between Sequence Variants and Chromatin Structure. Curr Genomics. 2010;11:359-67.

40. Wang S, Fu C, Zou Y, Wang H, Shi Y, Xu X, et al. Polymorphisms of angiotensin-converting enzyme 2 gene associated with magnitude of left ventricular hypertrophy in male patients with hypertrophic cardiomyopathy. Chin Med J (Engl). 2008;121:27-31.

41. Lieb W, Graf J, Götz A, König IR, Mayer B, Fischer M, et al. Association of angiotensin-converting enzyme 2 (ACE2) gene polymorphisms with parameters of left ventricular hypertrophy in men. Results of the MONICA Augsburg echocardiographic substudy. J Mol Med Berl Ger. 2006;84:88-96.

42. Nyati KK, Zaman MM-U, Sharma P, Kishimoto T. Arid5a, an RNA-Binding Protein in Immune Regulation: RNA Stability, Inflammation, and Autoimmunity. Trends Immunol. 2020;41:255-68.

43. Zhang Q, Cong M, Wang N, Li X, Zhang H, Zhang K, et al. Association of angiotensin-converting enzyme 2 gene polymorphism and enzymatic activity with essential hypertension in different gender. 
Medicine. 2018;97:e12917.

44. He J, Lu Y-P, Li J, Li T-Y, Chen X, Liang X-J, et al. Fetal But Not Maternal Angiotensin Converting Enzyme (ACE)-2 Gene Rs2074192 Polymorphism is Associated with Increased Risk of Being a Small For Gestational Age (SGA) Newborn. Kidney Blood Press Res. 2018;43:1596-606.

45. Cafiero C, Rosapepe F, Palmirotta R, Re A, Ottaiano MP, Benincasa G, et al. Angiotensin System Polymorphisms' in SARS-CoV-2 Positive Patients: Assessment Between Symptomatic and Asymptomatic Patients: A Pilot Study. Pharmacogenomics Pers Med. 2021;14:621-9.

\section{Tables}

Due to technical limitations, table 1 to 5 is only available as a download in the Supplemental Files section.

\section{Figures}

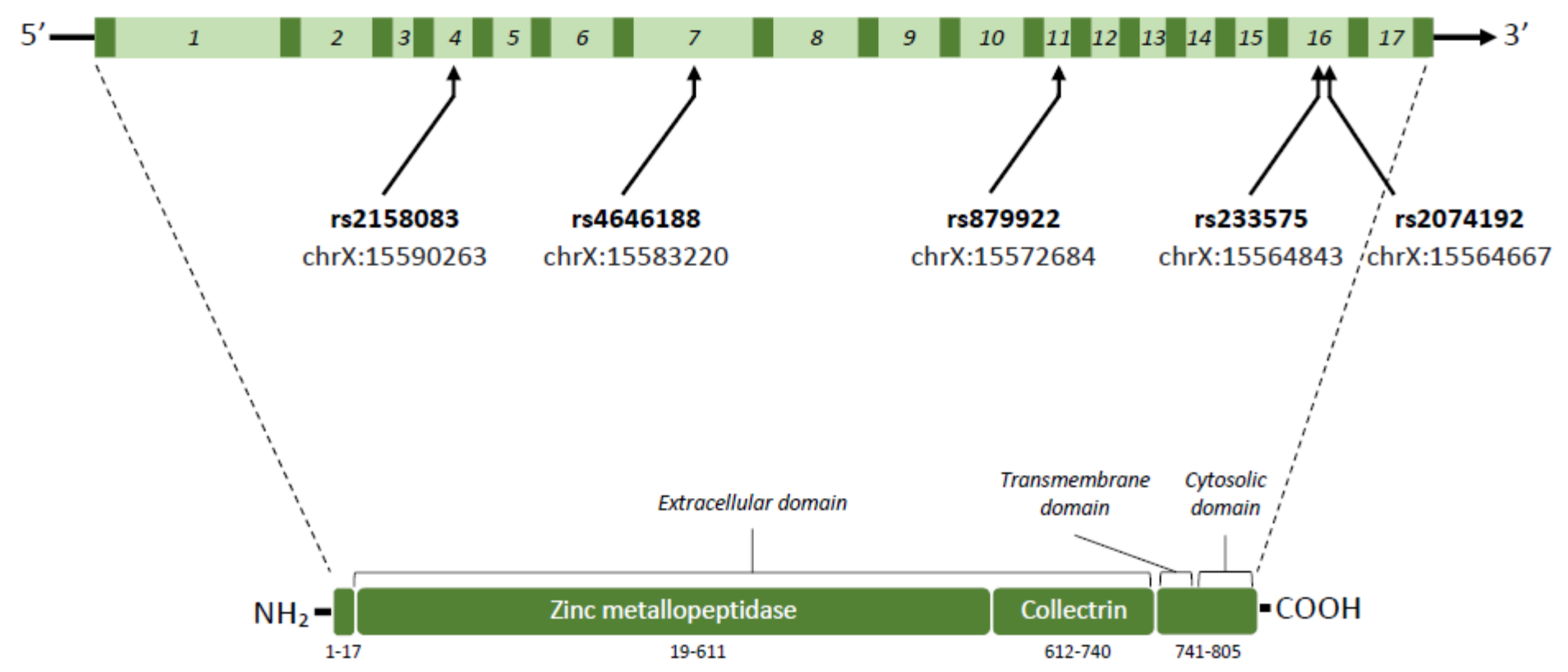

\section{Figure 1}

Schematic illustration of ACE2 gene and protein. The homo sapiens ACE2 gene (3,339 base pairs) is located on the chromosome X (Xp22.2; nucleotides 15,494,402-15,602,148; GRCh38.hg38 version). ACE2 comprises 18 exons and 17 introns (1-17 numbers) where the rs4646188, rs879922, rs233575, rs2074192, and rs2158083 SNPs can be found. After mRNA processing and splicing, the full length ACE2 protein includes 805 amino acids with different regions and domains [signal peptide (1-17 aa), Zincbinding metalloproteinase (19-611 aa), collectrin-like domain (612-740 aa), and C-terminal transmembrane anchoring region (741-805 aa)] (https://www.uniprot.org/uniprot/Q9BYF1). 


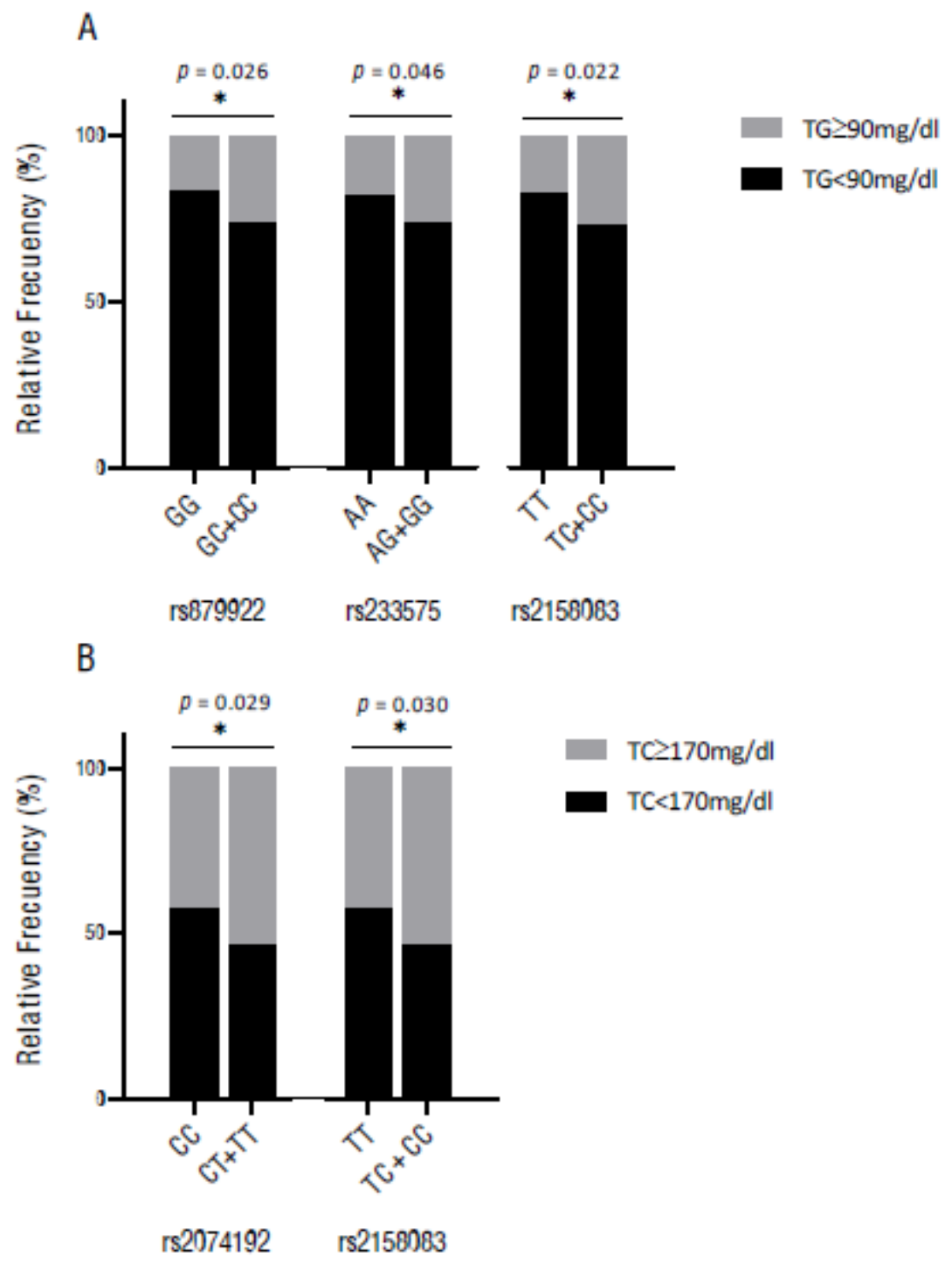

Figure 2

ACE2-SNPs and the risk of elevated TG and TC concentrations. Female adolescents were classified following the TG levels $\geq 90 \mathrm{mg} / \mathrm{dl}(\mathrm{A})$ or the TC levels $\geq 170 \mathrm{mg} / \mathrm{dl}(\mathrm{B})$, and the presence of ACE-SNPs was detected. ${ }^{\star} p<0.05$ minor vs. major allele.

\section{Supplementary Files}

This is a list of supplementary files associated with this preprint. Click to download.

- renamede77db.pdf 